\title{
Câncer e suicídio em idosos: determinantes psicossociais do risco, psicopatologia e oportunidades para prevenção
}

\author{
Cancer and suicide among the elderly: psychosocial determinants \\ of risks, psychopathology and opportunities for prevention
}

Manoel Antônio dos Santos ${ }^{1}$

${ }^{1}$ Departamento de Psicologia, Faculdade de Filosofia, Ciências e Letras de Ribeirão Preto, Universidade de São Paulo. Av. Bandeirantes 3900, Vila Monte Alegre. 14040-900 Ribeirão Preto SP Brasil. manoelmasantos@ gmail.com

\begin{abstract}
Suicide is a serious public health problem worldwide. Increasing age is directly associated with the rising rates of cancer and physical and functional limitations are important factors regarded as being associated with suicidal behavior among the elderly. This study sought to conduct a critical review of the literature on the risk factors associated with suicide among elderly cancer patients published between 2000 and 2015. Psychosocial precipitants of risks and psychopathology in 20 selected articles were conducted. The studies consistently identified a number of factors that have been considered to be associated with suicidal behavior among the elderly diagnosed with cancer. These include physical and mental health constraints (particularly major depression), social isolation, and the manner in which these factors and others interact. Further research is needed given the importance of the issue and to examine whether further education for healthcare providers and their abilities in suicide risk assessment and management could have positive effects on reducing the suicide rates among elderly patients with cancer. Considerations for upcoming studies encourage the adoption of empirically supported interventions for individualized management of the elderly cancer patient.
\end{abstract}

Key words Elderly, Neoplasms, Suicide, Risk factors, Review
Resumo O suicídio é um sério problema de saúde pública em todo o mundo. O avanço da idade está diretamente associado ao aumento da incidência de câncer e as limitações físicas e funcionais decorrentes da doença são fatores associados ao comportamento suicida em idosos. Este estudo teve por objetivo revisar criticamente a literatura sobre os fatores de risco associados ao suicídio em pacientes idosos com câncer, publicada entre 2000 e 2015. Foram examinados os determinantes psicossociais de risco e a psicopatologia em 20 artigos selecionados. Os estudos consistentemente identificam um conjunto de fatores que têm sido associados ao comportamento suicida em idosos com câncer, que incluem comprometimento físico e mental (particularmente depressão maior), isolamento social e o modo como esses fatores e outros interagem entre si. Dada a importância do tema, novas pesquisas se fazem necessárias para examinar se a educação do profissional de saúde e o incremento de suas habilidades de avaliação e manejo do risco suicida poderiam ter efeitos positivos na redução das taxas de suicídio observadas entre idosos com câncer. Considerações para estudos futuros encorajam a adoção de intervenções baseadas em evidências empíricas para o manejo individualizado do paciente oncológico idoso.

Palavras-chave Idoso, Neoplasias, Suicídio, Fatores de risco, Revisão 


\section{Introdução}

O suicídio é um grave problema de saúde pública em todo o mundo'. Para se ter uma noção da magnitude do problema, sua dimensão epidemiológica e relevância social, basta dizer que se trata da principal causa de morte violenta na população geral. A Organização Mundial de Saúde (OMS), em seu Relatório Mundial sobre Violência e Saúde, ratifica que as tentativas de suicídio na população idosa constituem um sério problema de saúde pública ${ }^{2}$. No marco da transição populacional e epidemiológica em marcha, e com o crescente aumento do contingente de idosos na população geral dos países desenvolvidos e em desenvolvimento, o suicídio passa a ser um problema cada vez mais agudo na agenda contemporânea dos países.

O segmento da população idosa, composto por indivíduos acima de 65 anos, está tendo um crescimento acelerado em virtude da alteração radical dos indicadores de mortalidade e natalidade $^{3}$. Segundo a OMS, as taxas populacionais, assim como as de morte autoinfligida, vêm crescendo em ritmo constante nesse grupo etário. $\mathrm{Na}$ América Latina, atualmente, a proporção de adultos em idade de trabalho, para cada pessoa com mais de 65 anos, é de 10:1, devendo cair para 3:1 em 20504. No Brasil, o crescimento exponencial do número de idosos representa um grande desafio para a saúde pública.

Assim como o suicídio, o câncer é uma importante causa de morte em todo o planeta e sua incidência está aumentando em escala global ${ }^{5}$. Vistas em seu conjunto, as projeções estatísticas permitem delinear uma população cada vez mais velha que é afetada pelo câncer, o que vai exigir do gestor de saúde políticas específicas, inclusive para os vários tipos de câncer mais prevalentes, como as neoplasias mamárias nas mulheres e o câncer de próstata nos homens ${ }^{6}$. Apesar da crescente incidência de câncer, que acompanha a tendência de envelhecimento da população, apenas uma minoria dos pacientes idosos tem acesso a benefícios como serviços de saúde de qualidade, inclusive para prevenção, informação e rastreamento clínico ${ }^{6}$.

O envelhecimento é o principal fator de risco para o indivíduo desenvolver câncer. O tratamento tem evoluído nas últimas décadas, porém também aumenta o risco de lesões e outras doenças, podendo afetar seriamente o bem-estar, a independência e o sentimento de autoestima do idoso. Essas são as questões cruciais que devem ser consideradas quando as decisões relativas ao tratamento oncológico estão sendo estabelecidas, bem como durante o seu andamento, que tende a ser prolongado e agressivo ${ }^{7}$.

O diagnóstico do câncer tem sido consistentemente associado a diversos transtornos mentais ${ }^{8}$ que podem potencializar as tendências autodestrutivas e, no limite, levar ao suicídio ${ }^{9}$. Será que pacientes idosos com câncer estariam mais propensos a cometer suicídio do que a população idosa sem câncer? A despeito da gravidade do problema ${ }^{10}$, há considerável lacuna de estudos que se ocupam da interface câncer e suicídio, o que pode alimentar a negligência de atenção dos profissionais de saúde para esse fenômeno, fazendo com que ele seja pouco reconhecido na prática clínica. Visando preencher essa necessidade, de modo a contribuir para a adoção de cuidados e estratégias de prevenção ao comportamento suicida em idosos com câncer, foi delineada a presente investigação.

Este estudo teve por objetivo revisar criticamente a literatura sobre os fatores de risco associados ao suicídio em pacientes idosos com câncer, examinando os determinantes psicossociais de risco e a psicopatologia.

\section{Método}

A fim de clarificar o estado da arte no tema do suicídio em pacientes geriátricos com câncer, realizamos um estudo de revisão integrativa da literatura publicada nos últimos anos. A questão de pesquisa que norteou esta revisão foi: "Quais são os fatores de risco associados ao suicídio em pacientes idosos com câncer?"

\section{Limites da busca}

Foram consultadas as seguintes bases indexadoras: PubMed, CINAHL, PsycINFO, Lilacs e SciELO. Os critérios de inclusão dos artigos foram: (1) período de publicação entre janeiro de 2000 e janeiro de 2015; (2) pacientes com 65 anos de idade e mais velhos; (3) artigos com foco específico sobre a questão do suicídio em pacientes oncológicos, independentemente do sexo, status socioeconômico e tipo de câncer; (4) artigos com um foco primário em resultados decorrentes de problemas emocionais e comportamentais, qualidade de vida ou ajustamento psicossocial, que podem potencializar o comportamento suicida; (5) artigos publicados nos idiomas inglês, espanhol e português.

Estudos que abrangeram grupos etários mais jovens foram incluídos apenas se incorporavam 
também a população idosa (por exemplo, estudos que analisavam os índices de suicídio em diferentes etapas do ciclo vital).

Critérios de exclusão: (1) artigos com um foco primário em resultados de tratamento oncológico; (2) artigos com foco principal nos cuidados paliativos e com pacientes em situação de terminalidade; (3) artigos com foco em assuntos que não estavam diretamente relacionados com o tema desta revisão, por discorrerem sobre autonegligências ou centrarem-se nas tentativas de suicídio e ideações suicidas.

Para nossos propósitos utilizamos várias combinações dos seguintes descritores: "idosos"/"elderly", "envelhecimento"/"aging”, "geriatria"/"geriatric", "câncer"/“cancer”, "suicídio"/“suicide”, "depressão”/“depression”, “ideação suicida"/"suicidal ideation", utilizando o operador booleano "and". Também foram realizadas buscas utilizando descritores não controlados, como "desesperança”/"hopelessness".

\section{Processo de seleção}

Os unitermos de pesquisa foram utilizados para extrair registros limitados à questão investigada. Um artigo foi considerado para inclusão no estudo se todos os critérios estabelecidos fossem satisfeitos.

Uma pesquisa adicional foi realizada para identificar estudos voltados especificamente para o suicídio em pessoas idosas com câncer. Nessa segunda etapa, optou-se por incluir artigos estritamente aderentes aos descritores "idoso", "neoplasia" e "suicídio". O critério da "adesão aos descritores" de um artigo foi definido como a presença de todas as supramencionadas palavraschave em qualquer parte do texto ou no abstract. Eventualmente, foram consideradas referências cruzadas e avaliação de artigos relatados nos diferentes textos recolhidos.

Desse modo, 3.716 artigos foram selecionados, combinando os descritores "suicídio" e "idosos", e 2.550 combinando "suicídio" e "câncer" nas bases PubMed, CINAHL, PsycINFO, Lilacs e SciELO. Desse total, foram inicialmente selecionados 110 artigos como potenciais estudos para avaliação, que preenchiam os critérios de limites de busca e seleção mencionados anteriormente. Além dos resumos, os textos foram recuperados em sua versão integral e lidos para extração das informações relevantes. Desse modo, foi possível operar um refinamento da busca. Depois de eliminadas as publicações duplicadas, isto é, encontradas em mais de uma base indexadora, ao final verificou-se que 20 dos artigos selecionados eram estritamente conectados com o tema dos fatores de risco para o suicídio em pacientes idosos com câncer, constituindo-se assim o corpus de análise (Quadro 1).

A revisão foi operacionalizada segundo critérios da análise documental ${ }^{4}$, que envolve: organização do material segundo os objetivos da revisão; análise da metodologia adotada nos artigos; abordagem do conteúdo das investigações e ênfase nos resultados e conclusões.

\section{Resultados}

- Transtorno depressivo e outras condições psiquiátricas

A despeito da alta prevalência de transtornos depressivos em pacientes idosos com câncer, essa temática permanece ainda pouco explorada. Uma revisão sistemática que investigou problemas de depressão, diagnóstico, patogênese, tratamento e suas interações neuroimunobiológicas ${ }^{10}$ evidenciou que a depressão em pacientes idosos com câncer pode assumir uma fenomenologia peculiar e ficar mascarada. Outra dificuldade encontrada é que a taxa moderada de transtorno depressivo maior e taxa elevada de transtorno depressivo menor por vezes são acompanhadas por formas subclínicas de depressão, que costumam ser subestimadas pelo profissional clínico. O humor disfuncional pode representar um substrato patogênico comum à depressão, ao câncer e ao processo de envelhecimento, o que pode ter implicações importantes para o tratamento. Nesse sentido, é preciso desenvolver métodos diagnósticos de transtorno de humor que sejam validados para a população idosa de cada país, de modo a permitir verificar a eficácia do tratamento antidepressivo para pacientes idosos com câncer, com o propósito de melhorar a evolução clínica e a qualidade de vida.

Embora estudos tenham demonstrado que em cerca da metade dos suicídios consumados por pacientes com câncer estava presente um quadro de depressão maior, há vários outros fatores relevantes que devem ser considerados, tais como a presença de ansiedade, a qualidade do apoio social e os sentimentos de impotência e desmoralização, que podem estar interligados com as manifestações de desesperança, angústia e solidão. É importante investigar a prevalência desses fatores psicológicos para ampliar o acervo de conhecimentos existentes acerca dos possíveis efeitos da depressão sobre o funcionamento imu- 
Quadro 1. Caracterização dos artigos recuperados segundo autores, ano e país da publicação, delineamento do estudo e principais resultados e conclusões encontrados $(n=20)$.

\begin{tabular}{|c|c|c|c|c|c|}
\hline No & Autores & Ano & País & $\begin{array}{c}\text { Delineamento } \\
\text { do estudo }\end{array}$ & Resultados e conclusões \\
\hline 10 & $\begin{array}{l}\text { Spoletini I, } \\
\text { Gianni W, } \\
\text { Repetto L, Bria } \\
\text { P, Caltagirone } \\
\text { C, Bossù P, } \\
\text { Spalletta G }\end{array}$ & 2008 & Itália & $\begin{array}{l}\text { Revisão } \\
\text { sistemática }\end{array}$ & $\begin{array}{l}\text { A elevada prevalência de transtornos depressivos } \\
\text { em pacientes idosos com câncer pode assumir } \\
\text { uma fenomenologia peculiar. Essa peculiaridade } \\
\text { pode contribuir para que a depressão permaneça } \\
\text { mascarada. }\end{array}$ \\
\hline 11 & $\begin{array}{l}\text { Rasic DT, Belik } \\
\text { SL, Bolton JM, } \\
\text { Chochinov HM, } \\
\text { Sareen J }\end{array}$ & 2008 & Canadá & $\begin{array}{l}\text { Estudo de } \\
\text { coorte }\end{array}$ & $\begin{array}{l}\text { Não foi encontrada associação entre câncer e } \\
\text { transtornos mentais na faixa etária mais avançada, } \\
\text { apesar de ter sido constatado aumento do risco de } \\
\text { suicídio. }\end{array}$ \\
\hline 12 & $\begin{array}{l}\text { Massie M, } \\
\text { Chertkov } \\
\text { L, Roth A. } \\
\text { Psychological } \\
\text { issues. In: } \\
\text { DeVita VJ, } \\
\text { Hellman S, } \\
\text { Rosenberg S }\end{array}$ & 2001 & EUA & $\begin{array}{l}\text { Revisão da } \\
\text { literatura }\end{array}$ & $\begin{array}{l}\text { Embora um número reduzido de pacientes com } \\
\text { câncer cometam suicídio, podem apresentar risco } \\
\text { um pouco maior do que a população em geral. } \\
\text { Pensamentos suicidas são relativamente comuns } \\
\text { enquanto o paciente luta contra uma doença com } \\
\text { elevado risco de morte e que o confronta com sua } \\
\text { condição de mortalidade. Fatores associados ao risco } \\
\text { aumentado de suicídio em pacientes com câncer } \\
\text { incluem: ser do sexo masculino, ter tumor associado } \\
\text { ao uso crônico e abusivo de álcool e tabaco, } \\
\text { estágio avançado da doença, pior prognóstico, } \\
\text { prejuízo no juízo crítico da realidade com pobre } \\
\text { controle de impulsos, não controle adequado da } \\
\text { dor, depressão, história de transtorno psiquátrico } \\
\text { ativo ou abuso anterior de substâncias psicoativas, } \\
\text { tentativas anteriores de suicídio, esgotamento físico } \\
\text { e emocional, e isolamento social. }\end{array}$ \\
\hline 13 & $\begin{array}{l}\text { Innos K, Rahu } \\
\text { K, Rahu M, } \\
\text { Baburin, A }\end{array}$ & 2003 & Estônia & $\begin{array}{l}\text { Estudo de } \\
\text { coorte }\end{array}$ & $\begin{array}{l}\text { Em uma coorte de } 65.419 \text { pessoas diagnosticadas } \\
\text { com câncer, foi encontrado aumento do risco de } \\
\text { suicídio para homens, mas não para mulheres. } \\
\text { Os homens tiveram o maior risco no intervalo de } \\
\text { tempo entre } 90 \text { e } 179 \text { dias após o diagnóstico. O } \\
\text { risco foi mais pronunciado para câncer de esôfago } \\
\text { e pâncreas. Há evidências de que o câncer é fator de } \\
\text { risco para suicídio pelo menos em homens. }\end{array}$ \\
\hline 14 & $\begin{array}{l}\text { Miccinesi G, } \\
\text { Crocetti E, } \\
\text { Benvenuti A, } \\
\text { Paci, E }\end{array}$ & 2004 & Itália & $\begin{array}{l}\text { Estudo de } \\
\text { coorte }\end{array}$ & $\begin{array}{l}\text { O estudo investigou se a mortalidade por suicídio } \\
\text { em pacientes com câncer durante o período 1985- } \\
1999 \text { diminuiu com o tempo em comparação } \\
\text { com a taxa observada na população geral. Foram } \\
\text { acompanhados } 90.197 \text { pacientes diagnosticasdos } \\
\text { com câncer, residentes na região central da Itália. } \\
\text { As taxas de mortalidade padronizadas foram } \\
\text { decrescentes durante os períodos avaliados, } \\
\text { indicando que a mortalidade por suicídio entre } \\
\text { pacientes com câncer declinou com o tempo em } \\
\text { relação à tendência de suicídio na população geral. }\end{array}$ \\
\hline
\end{tabular}

nológico e a progressão do câncer e, assim, delinear diretrizes específicas para o tratamento dos sintomas depressivos nessa população, visando beneficiar a qualidade de vida dos pacientes e de seus cuidadores ${ }^{10}$.
Estudo examinou a associação entre diagnóstico de câncer, transtornos mentais e pensamentos suicidas ${ }^{11}$. Entre os entrevistados com idades entre 55-75 anos, o câncer foi associado à suscetibilidade aumentada à agorafobia e dimi- 
Quadro 1. continuação

\begin{tabular}{|c|c|c|c|c|c|}
\hline No & Autores & Ano & País & $\begin{array}{c}\text { Delineamento } \\
\text { do estudo }\end{array}$ & Resultados e conclusões \\
\hline 15 & $\begin{array}{l}\text { Spoletini I, } \\
\text { Gianni W, } \\
\text { Caltagirone } \\
\text { C, Madaio } \\
\text { R, Repetto L, } \\
\text { Spalletta G }\end{array}$ & 2011 & Itália & $\begin{array}{l}\text { Revisão } \\
\text { sistemática }\end{array}$ & $\begin{array}{l}\text { A depressão em pacientes idosos com câncer } \\
\text { pode assumir uma fenomenologia peculiar. A } \\
\text { taxa moderada de transtorno depressivo maior } \\
\text { e a alta taxa de transtorno depressivo menor são } \\
\text { acompanhadas por formas subclínicas de depressão, } \\
\text { que frequentemente são subestimadas no contexto } \\
\text { assistencial. Na medida em que essas manifestações } \\
\text { não são reconhecidas pelos profissionais de } \\
\text { saúde, os pacientes não recebem tratamento. A } \\
\text { existência de uma possível disfunção imunológica } \\
\text { de base é hipotetizada como substrato patogênico } \\
\text { comum para problemas como depressão, câncer e } \\
\text { envelhecimento, o que pode ter implicações para o } \\
\text { tratamento. }\end{array}$ \\
\hline 16 & $\begin{array}{l}\text { Waern M, } \\
\text { Rubenowitz } \\
\text { E, Runeson } \\
\text { B, Skoog I, } \\
\text { Wilhelmson K, } \\
\text { Allebeck P }\end{array}$ & 2002 & Suécia & $\begin{array}{l}\text { Estudo } \\
\text { caso-controle }\end{array}$ & $\begin{array}{l}\text { Investigou-se a associação entre doenças físicas } \\
\text { e suicídio em pessoas idosas. A doença foi } \\
\text { determinada a partir de entrevistas com familiares } \\
\text { de pessoas que cometeram suicídio e registros } \\
\text { médicos consecutivos. Doença maligna, deficiência } \\
\text { visual e distúrbios neurológicos foram associados } \\
\text { a risco aumentado de suicídio em pessoas idosas. A } \\
\text { doença física grave e a elevada sobrecarga resultante } \\
\text { são fatores de risco mais decisivos para o suicídio } \\
\text { nos homens do que nas mulheres. }\end{array}$ \\
\hline 17 & $\begin{array}{l}\text { Miller M, } \\
\text { Mogun H, } \\
\text { Azrael D, } \\
\text { Hempstead K, } \\
\text { Solomon DH }\end{array}$ & 2008 & $\begin{array}{l}\text { Estados } \\
\text { Unidos }\end{array}$ & $\begin{array}{l}\text { Estudo } \\
\text { caso-controle }\end{array}$ & $\begin{array}{l}\text { Com o objetivo de determinar se o risco de suicídio } \\
\text { é maior entre os pacientes com câncer do que entre } \\
\text { aqueles que tinham outras doenças na população } \\
\text { idosa dos Estados Unidos. Os casos de pacientes } \\
\text { que morreram de } 1994 \text { a } 2002 \text { em consequência } \\
\text { de suicídio durante o período de estudo foram } \\
\text { pareados com pacientes de um grupo controle. } \\
\text { Na análise de regressão ajustada, a única condição } \\
\text { médica que permaneceu associada ao suicídio } \\
\text { foi o câncer. O suicídio também permaneceu } \\
\text { associado ao diagnóstico de transtorno afetivo e } \\
\text { de ansiedade, tratamento com antidepressivos e } \\
\text { analgésicos opiáceos. O risco de suicídio em adultos } \\
\text { idosos foi maior entre pacientes com câncer do que } \\
\text { em pacientes com outras doenças, mesmo após } \\
\text { contabilizar o diagnóstico psiquiátrico e o risco de } \\
\text { morte em um ano. }\end{array}$ \\
\hline
\end{tabular}

nuída a fobia social. O câncer não foi associado a qualquer transtorno mental no grupo acima de 75 anos. A ideação suicida foi associada com câncer no grupo etário de 55-74 anos, em modelos estatísticos não ajustados; no entanto, essa relação tornou-se não significativa quando foi feito o ajuste para as outras variáveis. Em síntese, os autores não encontraram associação entre câncer e transtornos mentais na faixa etária mais avançada, apesar de ter sido constatado aumento do risco de suicídio. Ainda assim, os pesquisadores ponderam que a depressão continua a ser importante fator de risco para o suicídio em pacientes oncológicos e preconizam que a triagem para transtornos depressivos e do pânico pode ser uma maneira clinicamente válida de identificar pacientes em risco.

O suicídio pode ser um meio de manter o senso de controle e uma alternativa reconfortante para pacientes que se sentem oprimidos pela 
Quadro 1. continuação

\begin{tabular}{|c|c|c|c|c|c|}
\hline No & Autores & Ano & País & $\begin{array}{c}\text { Delineamento } \\
\text { do estudo }\end{array}$ & Resultados e conclusões \\
\hline 18 & $\begin{array}{l}\text { Cole TB, } \\
\text { Bowling JM, } \\
\text { Patetta MJ, } \\
\text { Blazer DG }\end{array}$ & 2014 & $\begin{array}{l}\text { Estados } \\
\text { Unidos }\end{array}$ & $\begin{array}{l}\text { Estudo } \\
\text { caso-controle }\end{array}$ & $\begin{array}{l}\text { O estudo teve por objetivo determinar se o aumento } \\
\text { do risco de suicídio em pacientes com câncer pode } \\
\text { ser explicado pelas limitações funcionais, falta de } \\
\text { apoio social ou outros fatores relacionados. Após } \\
\text { ajuste para outros fatores de risco, houve associação } \\
\text { estatisticamente significativa entre suicídio e câncer, } \\
\text { mas não com ataque cardíaco, fratura de quadril } \\
\text { ou acidente vascular cerebral. O risco de suicídio } \\
\text { foi mais elevado para os homens versus mulheres, } \\
\text { brancos versus negros e pessoas com eventos vitais } \\
\text { estressantes ou limitações instrumentais, mas não } \\
\text { houve relação com atividades físicas de vida diária. } \\
\text { A probabilidade de suicídio declinou em indivíduos } \\
\text { casados, com cônjuge vivo versus outro status } \\
\text { marital, ou para aqueles que tinham um ou mais } \\
\text { indicadores de apoio social. }\end{array}$ \\
\hline 19 & $\begin{array}{l}\text { Björkenstam } \\
\text { C, Edberg A, } \\
\text { Ayoubi S, Rosén } \\
\text { M }\end{array}$ & 2005 & Suécia & $\begin{array}{l}\text { Estudo de } \\
\text { coorte }\end{array}$ & $\begin{array}{l}\text { A partir de uma coorte constituída por } 1.031 .919 \\
\text { pessoas diagnosticadas, as taxas de suicídio, } \\
\text { padronizadas para diferentes tipos de câncer, } \\
\text { foram analisadas por sexo ao longo de três décadas, } \\
\text { e cotejadas com as taxas de sobrevivência de } \\
\text { cinco anos. Foi comprovada a hipótese de que a } \\
\text { prevalência de suicídio é maior entre pacientes com } \\
\text { câncer do que na população geral. }\end{array}$ \\
\hline 20 & $\begin{array}{l}\text { Misono S, Weiss } \\
\text { NS, Fann JR, } \\
\text { Redman M, } \\
\text { Yueh B }\end{array}$ & 2008 & $\begin{array}{l}\text { Estados } \\
\text { Unidos }\end{array}$ & $\begin{array}{l}\text { Estudo de } \\
\text { coorte }\end{array}$ & $\begin{array}{l}\text { Partindo de uma coorte de } 3.594 .750 \\
\text { estadunidenses diagnosticados, verificou-se que o } \\
\text { aumento relativo do risco de suicídio em pacientes } \\
\text { oncológicos foi maior nos primeiros cinco anos após } \\
\text { o diagnóstico e declinou gradualmente depois desse } \\
\text { período crítico. No entanto, o risco permaneceu } \\
\text { elevado durante } 15 \text { anos depois do diagnóstico. } \\
\text { Taxas mais elevadas de suicídio entre pacientes com } \\
\text { câncer foram associadas ao sexo masculino, de cor } \\
\text { branca e solteiro. Ainda entre os homens, as taxas } \\
\text { de suicídio mais elevadas foram observadas com o } \\
\text { aumento da idade no momento do diagnóstico. Os } \\
\text { riscos mais elevados de suicídio foram detectados } \\
\text { em pacientes com câncer de pulmão e brônquio, } \\
\text { estômago, cavidade oral, faringe e laringe. }\end{array}$ \\
\hline 21 & Kendal WS & 2007 & Canadá & $\begin{array}{l}\text { Estudo de } \\
\text { coorte }\end{array}$ & $\begin{array}{l}\text { Estudo de base populacional analisou 1,3 milhões } \\
\text { de casos de câncer e comparou o risco de suicídio } \\
\text { entre os sexos. Pacientes de elevado risco para } \\
\text { suicídio eram do sexo masculino, com câncer de } \\
\text { cabeça e pescoço ou mieloma múltiplo, com doença } \\
\text { avançada, opções de tratamento limitadas e pouco } \\
\text { apoio social ou cultural. }\end{array}$ \\
\hline
\end{tabular}

continua

incerteza, sentimentos de impotência e temor de experimentar um sofrimento insuportável. A taxa real de suicídio em pacientes oncológicos é de difícil confirmação, pois os atestados de óbito podem refletir subnotificação de casos naqueles com doença avançada. O grau em que o aban- dono ou recusa do tratamento representa uma decisão deliberada do paciente de acabar com a própria vida ainda é desconhecido ${ }^{12}$.

Identificar e tratar a depressão em pacientes com câncer tem sido considerada uma estratégia efetiva para reduzir a morbidade e a mortalidade 
Quadro 1. continuação

\begin{tabular}{|c|c|c|c|c|c|}
\hline No & Autores & Ano & País & $\begin{array}{c}\text { Delineamento } \\
\text { do estudo }\end{array}$ & Resultados e conclusões \\
\hline 22 & $\begin{array}{l}\text { Lin } \mathrm{HC}, \mathrm{Wu} \\
\mathrm{CH}, \text { Lee HC }\end{array}$ & 2009 & Taiwan & $\begin{array}{l}\text { Estudo de } \\
\text { coorte }\end{array}$ & $\begin{array}{l}\text { O estudo verificou os fatores de risco associados } \\
\text { ao suicídio três meses após a alta hospitalar de } \\
\text { pacientes em tratamento oncológico em Taiwan. } \\
\text { Em torno de } 60 \% \text { dos óbitos por suicídio ocorreram } \\
\text { no primeiro mês após a alta. O intervalo médio de } \\
\text { consumação do suicídio foi de } 39,7 \text { dias da alta e } \\
\text { quase a metade ( } 46,3 \% \text { ) dos suicídios ocorreu no } \\
\text { intervalo de } 14 \text { dias pós-alta. O risco ajustado de } \\
\text { cometer suicídio para pacientes que não haviam } \\
\text { sido hospitalizados no ano anterior foi de } 1,68 \text { a } \\
2,51 \text { vezes maior do que os que foram internados no } \\
\text { ano anterior. O risco de suicídio entre os pacientes } \\
\text { desempregados foi } 1,71 \text { vezes maior do que entre } \\
\text { os empregados. Assim, os fatores de risco relevantes } \\
\text { incluem o número de hospitalizações no ano } \\
\text { anterior e a situação ocupacional. }\end{array}$ \\
\hline 23 & $\begin{array}{l}\text { Hann D, Baker } \\
\text { F, Denniston } \\
\text { M, Gesme D, } \\
\text { Reding D, Flynn } \\
\text { T, Kennedy J, } \\
\text { Kieltyka RL }\end{array}$ & 2002 & $\begin{array}{l}\text { Estados } \\
\text { Unidos }\end{array}$ & $\begin{array}{l}\text { Não } \\
\text { experimental, } \\
\text { descritivo e } \\
\text { correlacional }\end{array}$ & $\begin{array}{l}\text { Não foi confirmada a relação entre apoio social e } \\
\text { gravidade dos sintomas depressivos de acordo com a } \\
\text { idade e o sexo do paciente. } \\
\text { Uma rede de apoio social maior foi associada à } \\
\text { depressão menos grave para pacientes do sexo } \\
\text { feminino e os mais jovens, mas não para pacientes } \\
\text { do sexo masculino ou mais velhos. Para toda a } \\
\text { amostra (N = 342), maior adequação do apoio social } \\
\text { percebido e maior satisfação com o funcionamento } \\
\text { familiar foram relacionadas à depressão menos } \\
\text { grave. }\end{array}$ \\
\hline 24 & $\begin{array}{l}\text { Akechi T, } \\
\text { Nakano T, } \\
\text { Akizuki N, } \\
\text { Nakanishi T, } \\
\text { Yoshikawa E, } \\
\text { Okamura H, } \\
\text { Uchitomi Y }\end{array}$ & 2002 & Japão & $\begin{array}{l}\text { Não } \\
\text { experimental, } \\
\text { descritivo e } \\
\text { correlacional }\end{array}$ & $\begin{array}{l}\text { Estudo com pacientes japoneses internados em } \\
\text { uma unidade psiquiátrica de um hospital geral } \\
\text { verificou que 3,6\% das admissões foram motivadas } \\
\text { por questões relacionadas a suicídio, incluindo } \\
\text { casos com ideação suicida, tentativas de suicídio e } \\
\text { casos que haviam solicitado eutanásia e/ou sedação } \\
\text { contínua. A maioria dos pacientes apresentava } \\
\text { agravos físicos e/ou transtornos psiquiátricos. } \\
\text { Limitações no funcionamento físico e diagnóstico de } \\
\text { depressão maior foram os fatores clínicos associados } \\
\text { à conduta suicida em pacientes com câncer com } \\
\text { diferenças estatisticamente significantes. }\end{array}$ \\
\hline 25 & $\begin{array}{l}\text { Breitbart W, } \\
\text { Rosenfeld B, } \\
\text { Pessin H, Kaim } \\
\text { M, Funesti- } \\
\text { Esch J, Galietta } \\
\text { M, Nelson CJ, } \\
\text { Brescia R }\end{array}$ & 2000 & $\begin{array}{l}\text { Estados } \\
\text { Unidos }\end{array}$ & $\begin{array}{l}\text { Não } \\
\text { experimental, } \\
\text { descritivo e } \\
\text { correlacional }\end{array}$ & $\begin{array}{l}\text { Em uma amostra de } 92 \text { pacientes com câncer em } \\
\text { situação de terminalidade, } 17 \% \text { foram classificados } \\
\text { como tendo um desejo elevado de antecipar a } \\
\text { própria morte e } 16 \% \text { preencheram os critérios para } \\
\text { episódio depressivo atual. Desejo de morte acelerada } \\
\text { foi significativamente associado ao diagnóstico } \\
\text { clínico de depressão, bem como à gravidade dos } \\
\text { sintomas depressivos. Depressão e desesperança } \\
\text { ofereceram contribuições independentes e únicas } \\
\text { para a predição de desejo de morte acelerada, } \\
\text { enquanto que o apoio social e o funcionamento } \\
\text { físico mostraram contribuição adicional } \\
\text { significativa, porém de menor importância. }\end{array}$ \\
\hline
\end{tabular}


Quadro 1. continuação

\begin{tabular}{|c|c|c|c|c|c|}
\hline No & Autores & Ano & País & $\begin{array}{c}\text { Delineamento } \\
\text { do estudo }\end{array}$ & Resultados e conclusões \\
\hline 26 & $\begin{array}{l}\text { Filiberti A, } \\
\text { Ripamonti C }\end{array}$ & 2002 & Itália & $\begin{array}{l}\text { Revisão } \\
\text { sistemática }\end{array}$ & $\begin{array}{l}\text { Dor descontrolada e outros sintomas físicos, } \\
\text { sofrimento psíquico e angústia existencial são } \\
\text { fatores que incrementam a vulnerabilidade ao } \\
\text { suicídio em pacientes com câncer. }\end{array}$ \\
\hline 27 & $\begin{array}{l}\text { Schairer C, } \\
\text { Brown, LM, } \\
\text { Chen BE, } \\
\text { Howard R, } \\
\text { Lynch CF, } \\
\text { Hall P, Storm } \\
\text { H, Pukkala } \\
\text { E, Anderson } \\
\text { A, Kaijser M, } \\
\text { Andersson M, } \\
\text { Joensuu H, } \\
\text { Fosså SD, Ganz } \\
\text { PA, Travis LB }\end{array}$ & 2006 & $\begin{array}{l}\text { Estados } \\
\text { Unidos }\end{array}$ & $\begin{array}{l}\text { Estudo de } \\
\text { coorte }\end{array}$ & $\begin{array}{l}\text { O estudo quantificou o risco de suicídio entre } \\
723.810 \text { mulheres diagnosticadas com câncer } \\
\text { de mama nos Estados Unidos e Escandinávia } \\
\text { entre } 1953 \text { e } 2001 \text {, utilizando registros de base } \\
\text { populacional. Foi encontrada uma taxa de suicídio } \\
\text { em pacientes com câncer de mama equivalente a } \\
4,1 \text { por } 100.000 \text { pessoas/ano. O risco de suicídio se } \\
\text { mostrou elevado ao longo de todo o seguimento } \\
\text { e foi significativamente maior entre mulheres } \\
\text { negras. Houve aumento do risco com a progressão } \\
\text { do câncer de mama, mesmo decorridos } 30 \text { anos } \\
\text { do diagnóstico, evidenciando que os efeitos das } \\
\text { sequelas psicológicas podem perdurar no longo } \\
\text { prazo. }\end{array}$ \\
\hline 28 & $\begin{array}{l}\text { O’Mahony } \\
\text { S, Goulet J, } \\
\text { Kornblith A, } \\
\text { Abbatiello } \\
\text { G Clarke B, } \\
\text { Kless-Siegel S, } \\
\text { Breitbart W, } \\
\text { Payne R }\end{array}$ & 2005 & $\begin{array}{l}\text { Estados } \\
\text { Unidos }\end{array}$ & $\begin{array}{l}\text { Estudo } \\
\text { observacional }\end{array}$ & $\begin{array}{l}\text { Dos pacientes de Nova York internados para } \\
\text { controle da dor e cuidados paliativos, } 66 \% \text { não } \\
\text { expressavam desejo de morte antecipada no início } \\
\text { do estudo e } 45 \% \text { tiveram escores equivalentes à } \\
\text { depressão leve. Apenas } 40 \% \text { dos pacientes com } \\
\text { depressão moderada/grave estavam recebendo } \\
\text { antidepressivos. A melhora na depressão contribui } \\
\text { para moderar a gravidade do desejo de antecipação } \\
\text { da morte na população de pacientes com dor } \\
\text { decorrente do câncer. }\end{array}$ \\
\hline 33 & $\begin{array}{l}\text { Hem E, Loge } \\
\text { JH, Haldorsen T, } \\
\text { Ekeberg } \varnothing\end{array}$ & 2004 & Noruega & $\begin{array}{l}\text { Estudo de } \\
\text { coorte }\end{array}$ & $\begin{array}{l}\text { Estudo de coorte acompanhou } 490.245 \text { pacientes } \\
\text { oncológicos da Noruega entre } 1960 \text { e } 1999 \text {, dos } \\
\text { quais } 589 \text { pacientes ( } 407 \text { homens e } 182 \text { mulheres) } \\
\text { cometeram suicídio. O risco relativo foi elevado para } \\
\text { pacientes masculinos e femininos, especialmente } \\
\text { nos primeiros meses após o diagnóstico. Para } \\
\text { ambos os sexos, houve diminuição significativa do } \\
\text { risco de suicídio ao longo das décadas. O risco foi } \\
\text { significativamente maior entre pacientes masculinos } \\
\text { com neoplasias de órgãos respiratórios. }\end{array}$ \\
\hline
\end{tabular}

nos países ocidentais ${ }^{12-15}$. A identificação de pacientes com risco aumentado de cometer suicídio e o reconhecimento dos fatores biológicos e psicológicos subjacentes são o primeiro passo na prevenção, o que permite planejar intervenções em cuidados paliativos para pacientes potencialmente suicidas ${ }^{15}$.

As motivações que estariam por trás das altas taxas de suicídio frequentemente apontam para a depressão. É um dado bem estabelecido pela epidemiologia que a depressão é fator de risco para suicídio. Quase todos os estudos publicados sobre a questão da comorbidade psiquiátrica en- contraram alta incidência de transtornos de humor depressivo entre pacientes com câncer. Pesquisadores italianos advertem que, para além dos transtornos depressivos maior e menor, existe também elevada taxa de depressão menos grave em idosos com câncer que, muitas vezes, é subestimada e não tratada ${ }^{10}$.

Também há evidências recentes de que a vulnerabilidade à depressão pode não ser a única explicação para o fenômeno do suicídio em pacientes oncológicos. Sugere-se que a desesperança desempenha um papel possivelmente maior na determinação do comportamento suicida nesses 
pacientes e que limitações funcionais e falta de apoio social também contribuem para o aumento da taxa de suicídio entre pacientes idosos com câncer.

- Comparação com outras condições médicas Vários estudos do tipo caso-controle analisaram o risco de suicídio associado a doenças em pacientes com diferentes condições médicas e procedentes de diferentes regiões do planeta. Estudo caso-controle realizado na Suécia teve por objetivo investigar a associação entre doenças físicas e suicídio em pessoas idosas ${ }^{16}$. A doença foi determinada a partir de entrevistas com familiares de pessoas que cometeram suicídio e de registros médicos consecutivos (46 homens, 39 mulheres), pareados com participantes de controle (84 homens, 69 mulheres). A doença física grave foi a principal medida de desfecho. Doença maligna, deficiência visual e distúrbios neurológicos foram associados a risco aumentado de suicídio em pessoas idosas. Doença física grave em qualquer categoria foi um fator de risco independente para o suicídio no modelo de regressão multivariada. Na análise por sexo, doença física grave foi associada a suicídio nos homens e a carga de doença física foi elevada. Essas associações não foram encontradas em mulheres. Conclui-se que doença física grave e sobrecarga de cuidados são fatores de risco mais fortes para o suicídio em homens do que em mulheres.

Estudo caso-controle examinou o risco relativo de suicídio associado a uma variedade de condições médicas no contexto estadunidense ${ }^{17}$. Os resultados mostraram que a única condição médica que permaneceu associada ao suicídio foi o câncer. O risco de suicídio em idosos foi maior entre os pacientes com câncer do que entre aqueles que apresentavam outras condições médicas, mesmo quando contabilizados os transtornos psiquiátricos e o risco de morte em um ano. Essa conclusão explicita a carga peculiar que o diagnóstico de câncer impõe nos pacientes idosos, em virtude da estigmatização social da doença e de sua associação, no imaginário coletivo, com morte iminente ("sentenção de morte") e dolorosa.

Esse achado foi corroborado por outro estudo caso-controle, também conduzido com população dos EUA (Carolina do Norte), com vistas a investigar os fatores associados ao aumento do risco de suicídio em indivíduos com câncer ${ }^{18}$. O estudo buscou determinar se o aumento do risco de suicídio nesses idosos poderia ser explicado pelas limitações funcionais, falta de apoio social ou outros fatores relacionados. Os resultados fo- ram comparados com os de participantes idosos não acometidos por câncer. Quando realizado o ajuste para outros fatores de risco, encontrou-se associação estatisticamente significativa entre suicídio e câncer, mas não com ataque cardíaco, fratura de quadril ou acidente vascular cerebral, ou seja, com outras condições igualmente incapacitantes e potencialmente fatais. O risco de suicídio também foi elevado para os homens versus mulheres, brancos versus negros e pessoas submetidas a eventos vitais estressantes ou limitações instrumentais, mas não apresentou relação com limitações nas atividades físicas de vida diária, dificuldade respiratória ou má qualidade do sono. A probabilidade de suicídio foi significativamente menor em idosos casados com cônjuge vivo ou que contavam com um ou mais indicadores de apoio social.

Estudo sueco testou a hipótese de que o suicídio é mais prevalente entre pacientes com câncer do que na população geral e que essa associação muda ao longo do tempo; também investigou se o risco depende da gravidade da doença ${ }^{19}$. As taxas de suicídio padronizadas para diferentes tipos de câncer foram analisadas por sexo ao longo de três décadas, e cotejadas com as taxas de sobrevivência de cinco anos. Os resultados apontaram que pacientes com câncer apresentam maior risco de cometer suicídio do que a população geral.

- Fatores sociodemográficos

Estudo revelou que, na população geral dos Estados Unidos, a taxa de suicídio entre 19732002 foi de 31,4 por 100.000 pessoas-ano entre os pacientes com câncer, em comparação com 16,7 por 100.000 habitantes ao longo de um ano na população geral ${ }^{20}$. (No Brasil, o coeficiente de mortalidade por suicídio é, em média, 4,5 por 100.000 habitantes-ano.) Estudos anteriores, conduzidos principalmente na Europa, sugerem que pacientes com câncer podem estar em risco aumentado para o suicídio, mas esse foi o primeiro grande estudo de coorte realizado nos Estados Unidos comparando doentes oncológicos com a população geral. O aumento relativo do risco de suicídio foi maior nos primeiros cinco anos após o diagnóstico e declinou gradualmente após esse período crítico. No entanto, o risco permaneceu elevado durante 15 anos após o diagnóstico. Taxas mais elevadas de suicídio entre pacientes com câncer foram associadas ao sexo masculino, de cor branca e solteiro. Ainda entre homens, as taxas de suicídio mais elevadas foram observadas com o aumento da idade no momento do diagnóstico. 
Também realizado nos EUA, estudo casocontrole de base populacional ${ }^{18}$ mostrou que o risco relativo de suicídio é elevado para homens quando comparados às mulheres, brancos em relação a negros, e indivíduos com eventos de vida estressantes ou limitações instrumentais, mas não com limitações nas atividades físicas de vida diária.

Estudo de base populacional realizado no Canadá ${ }^{21}$ comparou o risco de suicídio entre os sexos masculino e feminino, com o propósito de elucidar as características específicas de cada sexo. Foram analisados 1,3 milhões de casos de câncer. Evidencou-se que os pacientes de alto risco para o suicídio eram do sexo masculino, diagnosticados com câncer de cabeça e pescoço ou mieloma múltiplo, com doença avançada, opções de tratamento limitadas e pouco apoio social ou cultural. Como implicação dos resultados para a prática, o estudo recomenda que os oncologistas e os demais profissionais de saúde devem estar cientes do potencial para o suicídio de pacientes com câncer e dos fatores de risco associados à morte autoinflingida.

Investigação conduzida por pesquisadores suecos ${ }^{19}$ mostrou que as taxas de suicídio foram padronizadas para diferentes tipos de câncer e analisadas por sexo e diferentes períodos. Assim, diferentemente de outros estudos ${ }^{18,20,21}$, uma taxa ligeiramente mais elevada para as mulheres foi observada no período 1965-1974.

Estudo de coorte verificou os fatores de risco associados ao suicídio três meses após a alta hospitalar de pacientes em tratamento oncológico em Taiwan ${ }^{22}$. A coorte foi composta por todos os pacientes com câncer que receberam alta dos hospitais no período de 2002-2004, inclusive aqueles que cometeram suicídio no prazo de 90 dias da alta $(n=311)$. Em torno de $60 \%$ dos óbitos por suicídio ocorreram no primeiro mês após a alta. O intervalo médio de consumação do suicídio foi de 39,7 dias da alta e quase a metade $(46,3 \%)$ ocorreu no intervalo de 14 dias pós -alta. O risco ajustado de cometer suicídio para pacientes que não haviam sido hospitalizados no ano anterior foi de 1,68, 1,61 e 2,51 vezes maior, respectivamente, do que os pacientes que foram internados uma vez, duas vezes e mais de duas vezes no ano anterior. O risco de suicídio entre os pacientes desempregados foi 1,71 vezes maior do que nos empregados. Assim, os fatores de risco relevantes incluem o número de hospitalizações no ano anterior e a situação ocupacional do paciente com câncer.
- Apoio social

A relação entre apoio social e sintomatologia depressiva em pacientes com câncer está bem estabelecida, porém a extensão em que as variáveis individuais impactam essa relação não é bem conhecida. A partir dessa premissa, um estudo foi delineado para verificar se a relação entre apoio social e gravidade dos sintomas depressivos varia de acordo com a idade e sexo dos pacientes ${ }^{22}$. A amostra foi constituída por 342 pacientes estadunidenses diagnosticados com câncer. Não houve diferenças significativas por sexo ou idade na relação das variáveis de apoio social com os sintomas depressivos. Foram encontradas diferenças interessantes, embora não estatisticamente significativas: uma rede de apoio social maior foi associado à depressão menos grave para pacientes do sexo feminino e para os mais jovens, mas não para pacientes do sexo masculino ou mais velhos. Para a totalidade da amostra, maior adequação do apoio social percebido e maior satisfação com o funcionamento familiar foram relacionadas à depressão menos grave. Os resultados sugerem que as intervenções propostas para aliviar os sintomas depressivos em pacientes com câncer devem considerar algumas características demográficas, como sexo e idade, e de apoio social para maximizar seu impacto benéfico na qualidade de vida.

Será que o aumento do risco de suicídio em indivíduos com câncer pode ser explicado pela insuficiência de apoio social? Para responder a essa pergunta foi conduzido um estudo casocontrole de base populacional, a partir de entrevistas com informantes primários para suicídio residentes na Carolina do Norte, Estados Uni$\operatorname{dos}^{18}$. Os resultados mostraram que o suicídio foi estatisticamente menos provável de ocorrer entre os participantes casados e com cônjuge vivo e naqueles que tinham um ou mais indicadores de apoio social.

O apoio social também parece ser uma variável importante na prevenção da morte autoinfligida, pois está associada a níveis menos graves de depressão para pacientes do sexo feminino e para os mais jovens, e com a vontade de viver ${ }^{23}$. Maior satisfação com o apoio social percebido também foi relacionada a sintomas de depressão menos graves. Mas foram constatadas diferenças e especificidades em termos do sexo e idade, que precisam ser melhor compreendidas por investigações futuras. 
- Tipo de câncer e local do acometimento

Estudo realizado nos $\mathrm{EUA}^{20}$ constatou que as taxas de suicídio variam de acordo com o tipo de câncer. As maiores taxas foram encontradas em pacientes com câncer de pulmão e brônquios, estômago, e cabeça e pescoço, incluindo cavidade oral, faringe e laringe. É reconhecido também que existe uma alta prevalência de depressão e/ou ansiedade entre os pacientes com esses tipos específicos de cânceres. O câncer de cabeça e pescoço, em particular, pode ter um efeito devastador na qualidade de vida dos pacientes devido às consequências sobre a aparência física (desfiguramento em uma área corporal de máxima visibilidade) e também em funções essenciais, como a fala, a deglutição e a respiração, o que pode explicar a elevada prevalência de autoextermínio nos pacientes acometidos por esses tipos de neoplasias.

- Grau de estadiamento do tumor

O câncer, quando detectado em estadiamento avançado, é uma doença com efeitos devastadores, que acarretam substancial estresse $\mathrm{e}$ sofrimento. Como mencionado anteriormente, o câncer tem sido consistentemente associado a sintomas psiquiátricos ${ }^{24}$, especialmente ao transtorno depressivo ${ }^{25} \mathrm{e}$ ao elevado risco de suicídio ${ }^{26}$. Esses agravos tendem a se acentuar na medida em que o prognóstico é reservado. Assim, a doença neoplásica avançada por si só constitui um fator que maximiza a vulnerabilidade ao estresse e ao sofrimento psicológico ${ }^{26}$, que por sua vez potencializa ou contribui para o comportamento suicida.

Estudo de coorte realizado na Suécia comprovou que o risco de suicídio em pacientes com câncer depende da gravidade da doença ${ }^{19}$. Houve ainda forte associação negativa entre as taxas de sobrevivência e as taxas de suicídio, ou seja, estas são mais elevadas nos casos de tumores graves, associados a baixas taxas de sobrevivência.

Estudo realizado com a população dos EUA comprovou que as taxas de suicídio foram maiores entre os pacientes com doença oncológica avançada no momento do diagnóstico ${ }^{20}$. Esse dado sugere a necessidade de redobrar a atenção nos momentos subsequentes à comunicação diagnóstica de um câncer metastático ou de tumores altamente agressivos. Estudo mostra que a investigação de possíveis pensamentos suicidas deve acompanhar sistematicamente as consultas, para que as medidas terapêuticas possam ser rapidamente instituídas quando necessárias ${ }^{26}$.

Compreender por que alguns pacientes com doenças terminais desejam acelerar a morte tor- nou-se uma questão importante em cuidados paliativos e no debate sobre a legalização do suicídio assistido. Estudo mostra que o desejo de abreviar a vida apresentado por pacientes com câncer em estado terminal não é incomum ${ }^{25}$. Depressão e desesperança são os mais fortes preditores do desejo de acelerar a morte nessa população. Intervenções psicológicas que abordem dimensões como depressão, desesperança e apoio social são necessárias no contexto dos cuidados paliativos, particularmente no que se refere ao enfrentamento do desejo de morte antecipada.

- Tempo de diagnóstico

Poucos estudos examinaram o risco de suicídio a longo prazo entre mulheres sobreviventes ao câncer de mama. Uma pesquisa quantificou o risco de suicídio entre 723.810 mulheres diagnosticadas com câncer de mama nos Estados Unidos e Escandinávia entre 1953 e 2001, utilizando registros de base populacional ${ }^{27}$. No total, 836 pacientes com neoplasia mamária haviam se suicidado (4,1 por 100.000 pessoas-ano). O risco se mostrou elevado ao longo de todo o seguimento, incluindo nos 25 ou mais anos após o diagnóstico, e foi significativamente maior entre as mulheres negras. Foi constatado aumento do risco de suicídio com a progressão do câncer de mama e a taxa se manteve elevada para as mulheres diagnosticadas entre 1990 e 2001. A probabilidade cumulativa de suicídio foi de $0,2 \%$ decorridos 30 anos do diagnóstico de câncer de mama, evidenciando que os efeitos das sequelas psicológicas perduram após três décadas do diagnóstico.

A relação entre condição física e tentativa de suicídio tem sido intensamente investigada nos últimos anos. O desejo de acelerar a morte é relatado na literatura como uma manifestação comum em pacientes com dor decorrente do câncer. Atualmente muito se avançou em termos de analgesia nos pacientes oncológicos. No entanto, não há atualmente evidências robustas que deem apoio à hipótese de que a melhora do controle da dor contribua para atenuar o desejo de morte. Não obstante, estudo evidenciou que a melhora nos sintomas depressivos e no controle da dor pode moderar o desejo de morte acelerada na população de pacientes oncológicos com dor ${ }^{28}$.

Nos estudos sobre suicídio na população geral, o comportamento suicida aparece como uma tentativa extrema que o indivíduo faz para escapar de estados de tensão insuportáveis, resultantes de uma inundação maciça de estressores tanto internos como externos. A extrema vulnerabilidade ao estresse pode ter causas orgânicas 
e psicológicas, de modo que os fatores de risco para suicídio na população geral parecem se situar na interconexão dos estressores físicos e psicológicos ${ }^{15}$. Pacientes com doenças orgânicas e/ou transtornos psiquiátricos têm elevado risco para planejar e cometer suicídio e os pacientes oncológicos, em particular, têm um risco significativamente aumentado. A mortalidade por suicídio em pacientes com neoplasia pode ser considerada um marco do impacto emocional de receber um diagnóstico de câncer ${ }^{14}$.

- Presença de comorbidades

A população idosa é vulnerável a inúmeras condições adversas de saúde, que podem anteceder e coexistir com o acometimento pelo câncer. Como resultado da presença de diversas comorbidades, os pacientes idosos devem tomar muitos medicamentos, fenômeno conhecido como polifarmácia. Uma proporção significativa terá problemas com os seus regimes de medicação já existentes no momento em que são diagnosticados com câncer ${ }^{29}$. Além da polifarmácia, a equipe da geriatria irá lidar com problemas como fraturas decorrentes de quedas e incontinência urinária, que depreciam a qualidade de vida e limitam a independência dos pacientes, podendo constituir fatores que contribuem para a conduta suicida.

A prevalência de comorbidades no paciente oncológico aumenta com a idade ${ }^{30}$. A avaliação e o controle das comorbidades são fundamentais porque elas estão associadas à redução da expectativa de vida e constituem um fator prognóstico independente para o desfecho do tratamento oncológico. Além disso, elas podem comprometer a tolerância à quimioterapia antineoplásica ${ }^{31}$.

- Tendências contraditórias observadas

Ainda que o câncer seja associado a um risco aumentado de suicídio e tentativa de suicídio, há relatos que contradizem essa tendência. Estudo evidenciou que a mortalidade por suicídio entre pacientes com câncer diminuiu na região central da Itália em relação à tendência de suicídio na população em geral ${ }^{14}$. Esse achado pode ser explicado pela melhora nas opções de tratamento e por uma melhor comunicação do diagnóstico. De fato, a implementação de programas de rastreamento de base populacional têm propiciado inovações nos tratamentos, com consequente aumento das taxas de sobrevida global. Também tem havido avanços na questão da comunicação do diagnóstico, o que pode favorecer uma reação mais positiva do paciente frente a notícia de uma doença oncológica.
Ao considerar esses aspectos, Spoletini et al. ${ }^{15}$ postulam que o suicídio em pacientes com câncer não é meramente uma reação secundária ao diagnóstico, mas está ligado a uma vulnerabilidade biopsicológica específica e acentuada ao estresse, devido a distúrbios imunológicos. Essa hipótese estende um modelo, inicialmente descrito para explicar o suicídio na população geral, para doenças físicas nas quais o sistema imunológico é alterado. Assim, segundo os referidos autores, esse modelo proporciona uma explicação mais detalhada acerca do porquê o câncer constitui um fator de risco para o suicídio.

- Limitações metodológicas dos estudos

Algumas considerações metodológicas podem ser levantadas a partir da análise dos estudos que buscam esclarecer as causas de suicídio na população oncológica, publicados nos últimos anos. Os desenhos de pesquisa são predominantemente de corte transversal. Estudos longitudinais são necessários para identificar com precisão os preditores de comportamento suicida. Além disso, há necessidade de mais estudos para esclarecer a natureza da relação complexa entre estresse, depressão, sistema imunológico e câncer ${ }^{32}$, e sua evolução ao longo do tratamento.

Também não foram encontrados estudos de meta-análise, combinando os resultados das diferentes pesquisas já realizadas sobre o suicídio em pacientes com câncer. Esse tipo de estudo é importante porque permite uma apreciação objetiva das evidências disponíveis ${ }^{15}$. Essas questões metodológicas limitam as conclusões dos estudos que se propõem a responder a pergunta: por que pacientes com câncer estão propensos a cometer suicídio? ${ }^{33}$

A maior parte dos estudos que abordam o tema suicídio em pacientes com câncer se restringe a doentes terminais ou pacientes internados, que podem representar um subgrupo particular. Pouco se conhece sobre pacientes não institucionalizados e as variáveis psicossociais também foram relativamente pouco estudadas, à exceção da condição de não ter parceiro afetivo. Outras fontes de apoio social, além de ter companheiro, não têm sido consideradas ${ }^{23}$, apesar do suporte social ser uma variável que tem se mostrado relevante e associada com o desejo de viver.

\section{Discussão}

Estudos realizados na Europa encontraram prevalência aumentada de suicídio em idosos com 
câncer ${ }^{13,14,19}$. Nos Estados Unidos, os pacientes com câncer têm quase o dobro da incidência de suicídio do que o observado na população geral, sendo que as taxas variam entre as diferentes localizações anatômicas do tumor ${ }^{20}$. Esses dados justificam uma análise mais aprofundada das vivências psicológicas de pacientes oncológicos, em especial de pacientes com certos tipos de câncer.

Apesar da alta prevalência de estresse em pacientes idosos com câncer, o tema ainda permanece pouco explorado, principalmente no cenário de países com menor nível de desenvolvimento. Não foram encontrados estudos em países latino-americanos, incluindo o Brasil, evidenciando a necessidade de maior investimento de pesquisas no contexto local.

Considerando-se esses limites, o presente estudo permitiu levantar importantes aspectos clínicos e psicológicos do risco suicida entre pacientes oncológicos. Pode-se depreender da literatura revisada que ainda não são claros os mecanismos que subjazem ao ato suicida em pacientes oncológicos. As alterações imunológicas presentes no câncer poderiam explicar as evidências que apoiam a associação entre depressão e suicídio nessa população, mas essa hipótese precisa ser fundamentada por pesquisas futuras ${ }^{15}$.

Verifica-se na literatura que o subgrupo de indivíduos com câncer que apresentam risco elevado de suicídio é constituído de pacientes masculinos, idosos, com um tempo de sobrevida inferior devido ao estadiamento avançado, resultando em significativo comprometimento físico e social. Além disso, os resultados de diversos estudos caso-controle evidenciaram que, uma vez realizado o ajuste para outros fatores de risco, a taxa de suicídio foi fortemente associada com o câncer, mas não com outras condições incapacitantes e que também eram potencialmente fatais ${ }^{18}$.

Também se constatou que é preciso considerar o tipo de neoplasia. O câncer de cabeça e pescoço está fortemente associado ao aumento do risco de suicídio, provavelmente porque esses pacientes enfrentam mais eventos estressores e, consequentemente, maior impacto em sua qualidade de vida. Esse incremento do estresse pode também estar relacionado ao significado psicológico e simbólico atribuído à doença e aos consideráveis prejuízos sociais, levando a uma suscetibilidade aumentada para depressão, desesperança e dor. Além disso, uma vez que o risco de suicídio é mais elevado logo após a comunicação do diagnóstico, a maneira de comunicar a notícia e os indicadores de prognóstico torna-se particularmente importante.
Os resultados dos estudos revisados apóiam a hipótese de que a gravidade do câncer aumenta o risco de suicídio. A implicação clínica desse achado é que maior atenção deve ser dada à situação psicológica, assegurando que os cuidados se estendam aos aspectos emocionais dos pacientes com câncer. Como o câncer é considerado, atualmente, uma doença crônica, os profissionais de saúde devem estar alertas para a presença de sintomas depressivos, inclusive os subclínicos, que não são de fácil detecção e que por isso mesmo exigem maior proximidade e aprofundamento do vínculo com o paciente. A tendência ao comportamento suicida deve ser acompanhada durante todo o curso da doença e não somente após o diagnóstico ${ }^{33}$.

Os estudos que têm examinado os fatores de risco para suicídio na população acometida pelo câncer destacam o quanto os profissionais de oncologia devem redobrar a atenção às questões psicossociais e de saúde mental de seus pacientes. Os dados sobre a incidência de suicídio apontam claramente para a necessidade de focalizar, durante a consulta, os problemas psiquiátricos, investigando sobretudo a possibilidade de depressão e ideação suicida como desfechos aos quais os pacientes oncológicos podem estar suscetíveis. Isso fica patente, por exemplo, em um estudo ${ }^{17}$ no qual os pesquisadores descobriram que o câncer era a única doença significativamente associada ao suicídio. No referido estudo, os fatores de saúde mental associados ao suicídio foram: transtorno afetivo, transtorno de ansiedade, tratamento com antidepressivos e com analgésicos/opióides.

Apesar da alta prevalência de transtornos depressivos em pacientes idosos com câncer, a temática da depressão ainda permanece pouco explorada. Reconhecer que pacientes oncológicos estão expostos a maior risco de suicídio é importante no contexto clínico. É preciso que os profissionais da equipe multiprofissional desenvolvam uma compreensão acerca do estado psicológico dos pacientes com câncer e interroguem, durante as consultas, sobre sintomas de depressão, ansiedade, estresse, questionando inclusive a ideação suicida. Essa preocupação é crucial para a identificação de casos em situação de risco, de modo que se possa iniciar o tratamento específico para os sintomas psiquiátricos ${ }^{34}$. Os resultados dos estudos revisados evidenciam, ainda, que se deve prestar atenção redobrada aos pacientes com quadros mais graves e com dor não controlada.

De fato, os estudos evidenciam a necessidade de manter rigoroso controle do estresse e da dor. O screening para depressão também é importante porque o quadro depressivo pode reduzir a mo- 
tivação do paciente para prosseguir com o plano terapêutico ${ }^{35}$, especialmente em um tratamento invasivo e penoso como o indicado para o câncer. A avaliação das condições emocionais e cognitivas, como depressão e alterações de memória, também é altamente recomendada porque elas podem interferir com a compreensão e a aceitação do plano terapêutico.

\section{Considerações finais}

É necessário investigar melhor a patogênese e as interações neuroimunobiológicas de condições complexas como câncer, depressão e envelhecimento, e desenvolver instrumentos validados para o diagnóstico dos transtornos de humor na velhice. Também é preciso verificar a eficácia do tratamento antidepressivo para pacientes oncológicos idosos com depressão, a fim de melhorar sua evolução clínica e qualidade de vida ${ }^{15}$.

O rastreamento rigoroso para a depressão em pacientes idosos com diagnóstico de câncer deve ser feito especialmente logo após a descoberta da doença e em momentos críticos do tratamento. Mas essa não é a única medida que deve ser tomada. As intervenções psicológicas que abordem a desesperança podem e devem proporcionar uma oportunidade para prevenir o suicídio nessa população. Nessa vertente, intervenções que incidem sobre questões existenciais e focalizam a atenuação dos pensamentos pessimistas, bem como as modalidades calcadas na espiritualidade, como aconselhamento espiritual e meditação, podem contribuir para atenuar os conflitos existenciais, levando à diminuição da desesperança e, por conseguinte, à prevenção do suicídio em pacientes oncológicos em situação de risco. Abordagens que utilizam técnicas de aconselhamento, que envolvem falar diretamente com os pacientes em risco aumentado para o suicídio, bem como com suas famílias, podem ser efetivas na redução do acesso aos métodos mais comuns utilizados para cometer suicídio.

Os resultados desta revisão apoiam a hipótese de que é fundamental a melhoria da comunicação equipe-paciente-família, a fim de que se possa fornecer adequada intervenção psicológica nas situações de crise. Também é necessário oferecer os cuidados centrados no paciente, ter familiaridade com os princípios da filosofia dos cuidados paliativos e uma eficaz educação para a morte e suicidologia. Um consistente trabalho em equipe pode contribuir para reduzir a necessidade de suicídio em pacientes com câncer e, provavelmente, seu desejo de acelerar a morte ${ }^{26}$. 


\section{Referências}

1. Hawton K, van Heeringen K. Suicide. Lancet 2009; 373(9672):1372-1381.

2. Krug EG, Dahlberg LL, Mercy JA, Zwi AB, Lozano R, editores. Relatório mundial sobre violência e saúde. Geneva: Organização Mundial da Saúde (OMS); 2002.

3. Brito F. Demografia: 2. Transição demográfica no Brasil; 2010. [acessado 2015 maio 15]. http://www.passeiweb. com/estudos/sala_de_aula/geografia/brasil_transicao_ demografica

4. Minayo MCS, Cavalcante FG. Suicide attempts among the elderly: a review of the literature (2002/2013). Cien Saude Colet 2015; 20(6):1751-1762.

5. World Health Organization (WHO). Global cancer rates could increase by 50\% to 15 million by 2020 . Geneva: WHO; 1999.

6. Lichtman SM. Guidelines for the treatment of elderly cancer patients. Cancer Control 2003; 10(6):445-453.

7. American Society of Clinical Oncology (ASCO). Age and cancer. [acessado 2015 maio 10]. http://www. cancer.net/navigating-cancer-care/older-adults/aging-and-cancer

8. Henriksson MM, Isometsa ET, Hietanen PS, et al. Mental disorders in cancer suicides. J Affect Disord 1995; 36(1-2):11-20.

9. Ripamonti C, Filiberti A, Totis A, De Conno F, Tamburini M. Suicide among patients with cancer cared for at home by palliative-care teams. Lancet 1999; 354(9193):1877-1878.

10. Spoletini I, Gianni W, Repetto L, Bria P, Caltagirone C, Bossù P, Spalletta G. Depression and cancer: an unexplored and unresolved emergent issue in elderly patients. Crit Rev Oncol Hematol 2008; 65(2):143-155.

11. Rasic DT, Belik SL, Bolton JM, Chochinov HM, Sareen J. Cancer, mental disorders, suicidal ideation and attempts in a large community sample. Psychooncology 2008; 17(7):660-667.

12. Massie M, Chertkov L, Roth A. Psychological issues. In: DeVita VJ, Hellman S, Rosenberg S, editors. Cancer: principles and practice of oncology. Philadelphia: Lippincott Williams \& Wilkins; 2001. p. 3058-3065.

13. Innos K, Rahu K, Rahu M, Baburin A. Suicides among cancer patients in Estonia: a population-based study. Eur J Cancer 2003; 39(15):2223-2228.

14. Miccinesi G, Crocetti E, Benvenuti A, Paci, E. Suicide mortality is decreasing among cancer patients in Central Italy. Eur J Cancer 2004; 40(7):1053-1057.

15. Spoletini I, Gianni W, Caltagirone C, Madaio R, Repetto L, Spalletta G. Suicide and cancer: where do we go from here? Crit Rev Oncol Hematol 2011; 78(3):206-219.

16. Waern M, Rubenowitz E, Runeson B, Skoog I, Wilhelmson K, Allebeck P. Burden of illness and suicide in elderly people: case-control study. BMJ 2002; 324:1355.

17. Miller M, Mogun H, Azrael D, Hempstead K, Solomon DH. Cancer and the risk of suicide in older Americans. J Clin Oncol 2008; 26(29):4720-4724.

18. Cole TB, Bowling JM, Patetta MJ, Blazer DG. Risk factors for suicide among older adults with cancer. Aging Ment Health 2014; 18(7):854-860.

19. Björkenstam C, Edberg A, Ayoubi S, Rosén M. Are cancer patients at higher suicide risk than the general population? Scand J Public Health 2005; 33(3):208-214.

20. Misono S, Weiss NS, Fann JR, Redman M, Yueh B. Incidence of suicide in persons with cancer. J Clin Oncol 2008; 26(29):4731-4738.
21. Kendal WS. Suicide and cancer: a gender-comparative study. Ann Oncol 2007; 18(2):381-387.

22. Lin $\mathrm{HC}, \mathrm{Wu} \mathrm{CH}$, Lee HC. Risk factors for suicide following hospital discharge among cancer patients. Psychooncology 2009; 18(10):1038-1044.

23. Hann D, Baker F, Denniston M, Gesme D, Reding D, Flynn T, Kennedy J, Kieltyka RL. The influence of social support on depressive symptoms in cancer patients: age and gender differences. J Psychosom Res 2002; 52(5):279-283.

24. Akechi T, Nakano T, Akizuki N, Nakanishi T, Yoshikawa E, Okamura H, Uchitomi Y. Clinical factors associated with suicidality in cancer patients. Jpn J Clin Oncol 2002; 32(12):506-511.

25. Breitbart W, Rosenfeld B, Pessin H, Kaim M, Funesti-Esch J, Galietta M, Nelson CJ, Brescia R. Depression, hopelessness, and desire for hastened death in terminally ill patients with cancer. JAMA 2000; 284(22):2907-2911.

26. Filiberti A, Ripamonti C. Suicide and suicidal thoughts in cancer patients. Tumori 2002; 88(3):193-199.

27. Schairer C, Brown, LM, Chen BE, Howard R, Lynch CF, Hall P, Storm H, Pukkala E, Anderson A, Kaijser M, Andersson M, Joensuu H, Fosså SD, Ganz PA, Travis LB. Suicide after breast cancer: an international population-based study of 723810 women. J Natl Cancer Inst 2006; 98(19):1416-1419.

28. O’Mahony S, Goulet J, Kornblith A, Abbatiello G, Clarke B, Kless-Siegel S, Breitbart W, Payne R. Desire for hastened death, cancer pain and depression: report of a longitudinal observational study. J Pain Symptom Manage 2005; 29(5):446-445.

29. Lees J, Chan A. Polypharmacy in elderly patients with cancer: clinical implications and management. Lancet Oncol 2011; 12(13):1249-1257.

30. Balducci L, Extermann, M. Management of cancer in the older person: A practical approach. The Oncologist 2000; 5(3):224-237.

31. Yancik R, Ries LA. Aging and cancer in America: demographic and epidemiologic perspectives. Hematol Oncol Clin North Am 2000; 14:17-24.

32. Reiche EM, Nunes SO, Morimoto HK. Stress, depression, the immune system, and cancer. Lancet Oncol 2004; 5(10):617-625.

33. Hem E, Loge JH, Haldorsen T, Ekeberg Ø. Suicide risk in cancer patients from 1960 to 1999. J Clin Oncol 2004; 22(20):4209-4216.

34. Sharma SP. High suicide rate among cancer patients fuels prevention discussions. J Natl Cancer Inst 2008; 100(24):1750-1752.

35. Lyness JM, Noel TK, Cox C, King DA, Conwell Y, Caine ED. Screening for depression in elderly primary care patients: a comparison of the center for Epidemiologic Studies Depression Scale and the Geriatric Depression Scale. Arch Intern Med 1997; 157(4):449-454.

Artigo apresentado em 07/09/2015

Aprovado em 24/05/2016

Versão final apresentada em 26/05/2016 
